\section{Palatal stiffening via transoral, retrograde interstitial laser coagulation}

\author{
Yosef P. Krespi, ${ }^{1}$ Victor Kizhner ${ }^{2}$ \\ ${ }^{1}$ Center for Sleep Disorders, New York \\ Head Neck Institute; \\ ${ }^{2}$ Department of Otolaryngology \\ St.Luke's-Roosevelt Hospital Center, \\ New York, NY, USA
}

\begin{abstract}
Current treatment modalities for snoring may include mucosal removal, coblation or radiofrequency palatoplasty, injection snoreplasty and placement of palatal implants with described disadvantages. We introduce a new laser assisted method avoiding intraoral injury. A pilot study treating 13 loud snorers having an $\mathrm{RDI}<8$ was conducted. A diode laser coupled to a flexible fiberand a handle with curved needle was used. The fiber was introduced into the nasal surface of soft palate between palatoglossal and glossopharyngeal arches and advanced progressively anteriorly after pulling the uvula forward three times to create palatal scarring and stiffening. All responded to a phone survey. Six patients reported significant improvement, 4 had some improvement, 2 had mild improvement and one patient had no change. Pain score was moderate for 3 patients while the rest had mild pain. The laser harbors many advantages over other methods. Results with this technique are encouraging further studies.
\end{abstract}

\section{Introduction}

Snoring affects as high as $20 \%$ of the population and may impact breathing during sleep. Surgical and non surgical treatment options for snoring have soared in the past years especially with the advent of minimally invasive office procedures such as laser palatoplasty, radiofrequency (RF) volumetric reduction, the Pillar procedure and increase use oral appliances. Uvulo-palatopharyngoplasty, usually performed under general anesthesia will eliminate snoring with a significant amount of post-operative pain and is usually considered overkill in the absence of obstructive sleep apnea (OSA). ${ }^{1}$ Laser assisted uvulo-palatoplasty (LAUP) can be performed under local anesthesia in the office, also resulting in significant postoperative discomfort. The patient expectations from an elective snoring therapy without the presence of OSA are very high.
Patient satisfaction is greatest with an effective, rapid in-office procedure with minimal post surgical down time at a reasonable cost. ${ }^{2}$ Energy deliverance by an ablative mode using lasers or RF attempts to reduce excessive palatal volume with further scar formation, results in intrapalatal stiffening. RF has its drawbacks such as cost for disposables, the need for occasional repeated procedure due to absence of standardization, lack of uvular management, inability of energy control, complications (i.e., erosion and ulceration). A recent study (contrary to previous short term results) suggests that radiofrequency may be redundant for a long term desired effect. ${ }^{3,4}$

Energy deliverance by laser can achieve similar results and also being cost effective. Lasers are commonly found in ENT office practice used for wide range of applications. A 980nm near-infra-range (NIR) diode laser (A.R.C. laser, Nürnberg, Germany) is a small, economical, battery operated with a flexible fiber that can be attached to a hand-piece with curved needle tip. Energy deliverance at 7-8 watts is used in continuous mode in contact or interstitially by penetrating into the palatal muscles. The laser created less tissue necrosis and less thermal damage compared to monopolar cautery. ${ }^{2}$

We are presenting a new, minimally invasive, effective method for snoring cessation with a $980 \mathrm{~nm}$ NIR diode laser by targeting the nasal surface of the uvula and soft palate. One of the limitations of the previous surgical procedures is significant discomfort and swelling that will affect oral intake. By avoiding injury to the oral mucosa traversing and targeting the palatal muscles from its nasal surface we have avoided injury to oral mucosa and eliminated postoperative discomfort associated with deglutition.

\section{Materials and Methods}

Following injection of local anesthesia containing $1 \%$ lidocaine with epinephrine to the center soft palate in an office ENT chair, a 980nm NIR laser with 300micron fiber coupled to a surgical hand-piece and curved needle tip was used. Laser settings were 7-8W in continuous mode. Ten otherwise healthy snorers with $\mathrm{AHI}<10$ and no other co morbidities were treated in the office. The tip of the uvula was grasped gently with forceps and flipped anteriorly (Figure 1). The laser fiber was inserted penetrating the nasal surface of palatal mucosa around the base of the uvula and advanced further anteriorly into the soft palate muscles (Figure 2). At least three passes were made (one center and two lateral) for about $10 \mathrm{~s}$. The laser fiber was advanced in a retrograde fashion about $2-3 \mathrm{~cm}$ from the base
Correspondence: Victor Kizhner, 425 W 59st. 10th floor, New York, NY 10019, USA.

Tel. +1.212.262.4444 - Fax: +1.212.523.6364.

E-mail: vkizhner@gmail.com

Key words: snoring, palatal stiffening, diode laser.

Received for publication: 23 June 2011. Accepted for publication: 19 September 2011.

This work is licensed under a Creative Commons Attribution NonCommercial 3.0 License (CC BYNC 3.0).

(C) Copyright .P. Krespi and V. Kizhner, 2011

Licensee PAGEPress, Italy

Surgical Techniques Development 2011; 1:e23 doi:10.4081/std.2011.e23

of the uvula in the midline and two additional lateral passes were made (Figure 3). Total energy deliverance was no more than $1000 \mathrm{j}$. The patients filled a 0 -10VAS questionnaire where 0 is no snoring and 10 represents very loud and disturbing snoring. Results before and one month after the procedure were recorded. Complications if arose were noted. Pain intensity was collected based on scale comprised of mild / moderate or severe for simplification (Figure 4).

\section{Results}

All patients responded to a phone survey one month after the procedure. Six patients reported significant improvement, 4 had some improvement, 2 had mild improvement and one patient had no change. Pain score was moderate for 3 patients while the rest had mild pain. The follow up for 10 patients is now over a year with sustained results.

\section{Discussion}

We conducted a pilot study examining a new potential treatment for simple snoring without OSA. Our method is innovative by its retrograde technique and by keeping the oral mucosa intact. This is reflected in a posterior to anterior interstitial passage of the laser fiber. Our goal was to avoid damage or injury to oral mucosa by its preservation, allowing better oral intake, reduce postoperative pain and avoiding possible wound contamination. This method addresses palatal snoring via interstitial retrograde palatal laser stiffening. The laser achieves precise energy deliverance 
with an easy to use ergonomic hand-piece design. The laser wavelength combined with heat is ideal for interstitial coagulation resulting in stiffening. The lack of complications speculatively can be attributed to the laser's disinfectant properties. ${ }^{5}$

Histopathological comparison between RF and diode laser in the pig's nasal mucosa showed included necrosis and ulceration, fibrin deposition in the mucosa, necrotizing sialometaplasia, as well as proliferation of the granulation tissue for both modalities. As total energy deliverance for each modality is lacking it is hard to apply equal comparison. ${ }^{6}$

Patient satisfaction was high as their oral and pharyngeal pain was minimal and did not interfere with oral intake. Surgical time was short and done under local anesthesia in the

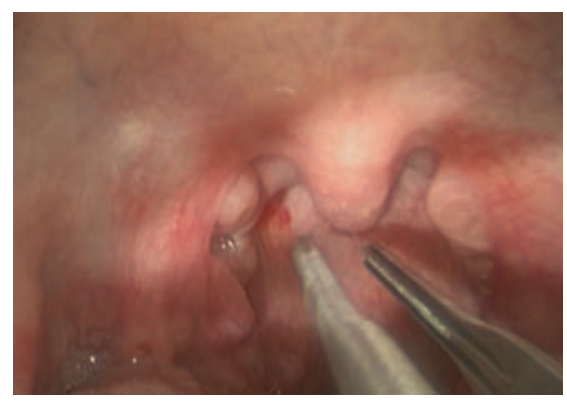

Figure 1. The uvula is flipped upwards.

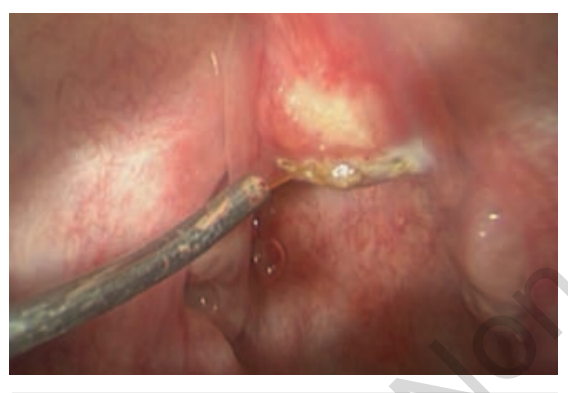

Figure 3. Retrograde interstitial laser ablation on pharyngeal side. office enabling quick recovery. As the proximal part of the fiber can be resterilized and reused, cost effectiveness is maintained. Current follow up for 10 patients is now a year with sustained results. This may predict promising long term results but with a small cohort and eliminating additional factors (such as weight gain) this task may be impossible.

This pilot study has several limitations. The follow up is not long enough and our cohort is small. Exact energy may be optimized better. Nevertheless a postero-anterior transnasal mucosa based interstitial ablation of the center compartment of soft palate performed in the office defines most of the factors qualifying for the ideal snoring treatment method.

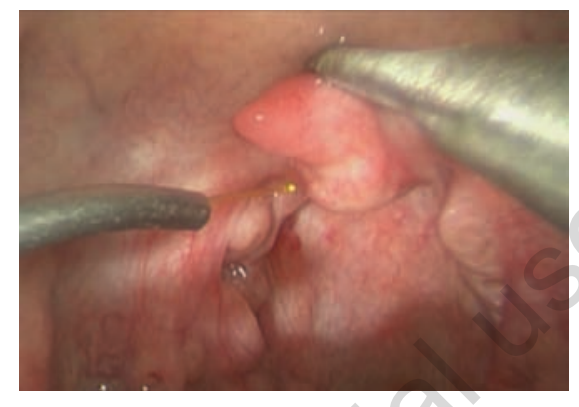

Figure 2. The laser is about to be introduced retrogradely in the flipped uvula.

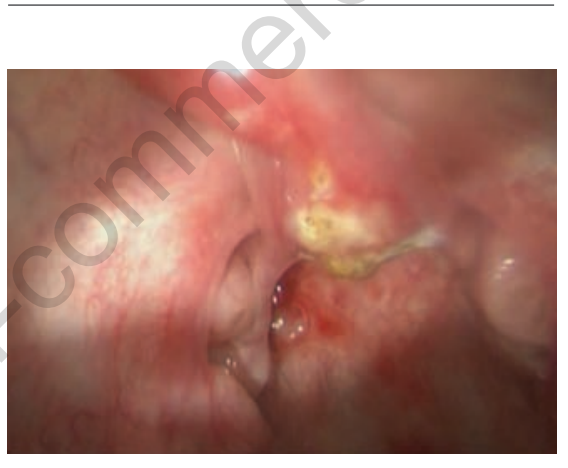

Figure 4. The result in the end.

\section{Conclusions}

We report encouraging results of this small series. The excellent wavelength absorption makes it ideal for tissue modulating without significant bleeding. We find it advantageous for the following reasons: inexpensive, reproducible results, minimal postoperative discomfort, can be energy titrated, pain is minimal, has possible favorable oral intake due to minimal interference with food passage, safe office procedure and patient appealing. Those results encourage further studies

\section{References}

1. Main C, Liu Z, Welch K, et al. Surgical procedures and non-surgical devices for the management of non-apnoeic snoring: a systematic review of clinical effects and associated treatment costs. Health Technol Assess 2009;13:iii, xi-xiv, 1-208.

2. Oswal V, Remacle M, Jovanvic S, Krespi J, eds. Principles and Practice of Lasers in Otorhinolaryngology and Head and Neck Surgery. The Hague, Netherlands: Kugler Publications; 2002.

3. Stuck BA. Radiofrequency-assisted uvulopalatoplasty for snoring: Long-term follow-up. Laryngoscope 2009;119:1617-20.

4. Bäck LJ, Hytönen ML, Roine RP, Malmivaara A0. Radiofrequency ablation treatment of soft palate for patients with snoring: a systematic review of effectiveness and adverse effects. Laryngoscope 2009;119:1241-50.

5. Kreisler M, Kohnen W, Marinello C, et al. Antimicrobial efficacy of semiconductor laser irradiation on implant surfaces. Int $\mathrm{J}$ Oral Maxillofac Implants 2003;18:706-11.

6. Zborayová K, Ryska A, Lánsky M, et al. Histomorphologic study of nasal turbinates after surgical treatment: a comparison of laser surgery and radiofrequencyinduced thermotherapy effects in animals. Acta Otolaryngol 2009;129:550-5. 